\title{
Agrobiodiversität, das Gemeinschaftserbe-Prinzip und Marktanreize
}

\author{
Cristian Timmermann und Zoë Robaey
}

cristian.timmermann@gmail.com

Dieser Text ist eine pre-print Version des folgenden Buchkapitels:

Timmermann, Cristian and Zoë Robaey (2016), "Agrobiodiversität, das Gemeinschaftserbe-Prinzip und Marktanreize". In Biopatente - Saatgut als Ware und als öffentliches Gut, Hrsg. von Barbara Brandl und Stephan Schleissing, 109-132. BadenBaden: Nomos.

Bitte zitieren Sie die Version des Verlags:

http://dx.doi.org/10.5771/9783845275246-108 


\title{
Agrobiodiversität, das Gemeinschaftserbe-Prinzip und Marktanreize
}

\author{
Cristian Timmermann und Zoë Robaey
}

\section{Einleitung: Agrobiodiversität fördern - warum?}

Die Diversität von Nahrungspflanzen, ein Ergebnis jahrtausendelanger Zuchtbemühungen, ist in den letzten Jahrzehnten dramatisch zurückgegangen. Schätzungen zufolge machen von den über 7000 Nahrungspflanzenarten ganze 103 Sorten 90\% der Nahrungsmittelproduktion aus. ${ }^{1}$ Dieser Verlust könnte in Zukunft gewaltige negative Auswirkungen auf die Nahrungsmittelsicherheit haben, da die Biodiversität eine zentrale Rolle bei der Absorbierung biotischer und abiotischer Stressfaktoren spielt, die auf die Pflanzen wirken. Darüber hinaus stellt der Verlust eine bedeutende Verarmung nicht nur des Pools genetischer Ressourcen dar, die zukünftigen Generationen zur Verfügung stehen, sondern auch der kulturellen Diversität, indem die Nahrungsmittelvielfalt der Landesküchen eingeschränkt und sowohl Kulturlandschaften als auch Stadtgärten vereinheitlicht werden. Wegen der grundlegenden Funktion, die die Agrobiodiversität in der menschlichen Gesellschaft erfüllt, werden wir im Folgenden verschiedene Schwierigkeiten bei der Pflege der Agrobiodiversität als ein gemeinsames Erbe der Menschheit in einer stark ungleichen Welt erörtern. Zuvor jedoch möchten wir untersuchen, was Agrobiodiversität eigentlich ist und welche Funktion sie für das menschliche Wohlbefinden erfüllt. Ziel dieses Artikels ist zu zeigen, inwieweit sich verschiedene Anreizsysteme unterschiedlich auf den Erhalt von Agrobiodiversität auswirken.

Was genau meint Agrobiodiversität? Der Begriff ist schwer einzugrenzen, da schon bei der Definition des zugrunde liegenden Konzepts der Biodiversität zahlreiche Schwierigkeiten auftreten. ${ }^{2}$ Zunächst einmal lässt sich schwer bestimmen, welche Organismen als lebend gelten (d. h. als Teil der Biosphäre) und in welchem Maß sie für die Nahrungsmittelproduktion relevant sind. Auch sind die Grenzen zwischen Kultursorten und halbwilden oder wilden Sorten oft genug nicht eindeutig, was die taxonomische Einordnung erschwert. In diesem Artikel haben wir mit der Verwendung des Begriffs Agrobiodiversität jene Nahrungspflanzensorten im Sinne, die für die Nahrungsmittelproduktion von direkter Bedeutung sind und die ihre heutige Form weitgehend bewussten menschlichen Bemühungen verdanken. Wir werden dabei einige direkte Vergleiche zur kulturellen Vielfalt ziehen, insofern zur Agrobiodiversität die willkürliche und zielgerichtete Auswahl und Zucht gehört. ${ }^{3}$

Trotz ihrer anerkannten Rolle für die zukünftige Nahrungsmittelsicherheit ist die Erhaltung der Agrobiodiversität nach wie vor eine komplexe Aufgabe, die eine dauerhafte Lösung benötigt. Betrachtet die globale Bevölkerung Biodiversität als öffentliches Gut, dann sieht sie sich mit folgenden drei Problemen konfrontiert: Erstens haben eine Reihe von Gemeinschaften über mehrere Jahrhunderte hinweg zur Existenz und Erhaltung des Gutes beigetragen; individuelle Beiträge sind daher unmöglich oder schwierig nachzuverfolgen. ${ }^{4}$ Zweitens haben einzelne Landwirte, die sich für Monokulturen entschieden haben, als Kollektiv zur allgemeinen genetischen Erosion beigetragen. ${ }^{5}$ Drittens besteht für kommerzielle

\footnotetext{
Tomich et al. 2011, 200

vgl. Malaterre 2013

vgl. Heyd 2010

Kloppenburg/Kleinman 1987

Lacy 1994
} 
Pflanzenzüchter die Möglichkeit, sich exklusive Rechte für ihre neuen Saatgutsorten zu sichern, auch wenn sie sich dafür genetischen Materials bedienen, das öffentlich zugänglich ist. ${ }^{6}$

Jedes Anreizsystem, das die Agrobiodiversität erhalten oder fördern soll, muss diese drei Facetten des öffentlichen Guts und die einhergehenden Probleme berücksichtigen und gleichzeitig auch anerkennen, dass der Agrobiodiversität auch unter dem Aspekt des Funktionierens eines Ökosystems sowie als Element des kulturellen Erbes ein Wert beigemessen werden muss.

\section{Agrobiodiversität: von der Nahrungsmittelsicherheit zum kulturellen Erbe}

Es gibt zwei große Argumentationsansätze zur Begründung der Bedeutsamkeit der Agrobiodiversität. Der eine konzentriert sich auf den instrumentellen Wert der verschiedenen Ökosystemleistungen, die eine solche Vielfalt erbringt. Der zweite Argumentationsansatz hingegen will die Agrobiodiversität ebenfalls aufgrund ihres intrinsischen Wertes als wesentliches Element der kulinarischen Kulturvielfalt der Welt sowie als ikonisches Merkmal der Kulturlandschaften verteidigen, also wegen ihrer »breiteren« Rolle als gemeinsames Erbe der Menschheit.

Mit welcher Argumentation die Naturschutzpolitik für den Erhalt der Agrobiodiversität eintritt, wird sich deutlich auf die Entscheidung auswirken, welche Sorten bestehen bleiben sollen. Diese Problemstellung spielt eine wichtige Rolle in der Debatte um anthropozentrisch oder ökozentrisch fundierte Erhaltungsmaßnahmen. ${ }^{7}$ Je nach den Motiven und Bedürfnissen der Menschen wird durch Schutzprogramme jeweils eine unterschiedliche Auswahl an Nahrungspflanzen erhalten bleiben. Ärmere Gesellschaften, in denen die Nahrungsmittelsicherheit ein größeres Problem darstellt, die aber industrialisiert genug sind, um sich eine Grundinfrastruktur für Agrarforschung zu leisten, werden ihre Schutzbemühungen weitestgehend auf ertragreiche Sorten von Nahrungspflanzen konzentrieren, die (für sich allein oder zusammen mit anderen Arten) bedeutenden Umweltstressfaktoren standhalten können. Wohlhabendere Länder dagegen sind in dieser Hinsicht deutlich flexibler und können Sorten in ihre Schutzprogramme mit aufnehmen, die überwiegend wegen ihres Geschmacks und Aussehens oder sogar als Tierfutter geschätzt werden, um ihr kulinarisches Erbe zu erhalten und zu erweitern.

\subsection{Agrobiodiversität und ihr Naturhaushaltswert}

Insbesondere Agrarökologen unterstreichen die zentrale Bedeutung der Biodiversität für die Gesundheit eines Ökosystems. Wie bereits erwähnt, verbessert die Sicherstellung der Artenvielfalt - darunter auch die Vielfalt an essbaren Pflanzen - die Fähigkeit eines Ökosystems, sowohl biotischen als auch abiotischen Stress zu absorbieren. ${ }^{8}$ Angesichts der Tatsache, dass der Klimawandel das Problem der Nahrungsmittelsicherheit weiter verschärft, indem er den Grad der Bodenversalzung, Temperaturen sowie das Ausmaß von Dürren und Regenfällen verändert und die Wanderung von Schädlingen in zuvor nicht betroffene Gebiete erleichtert, ${ }^{9}$ sind die Entwicklung neuer Nahrungspflanzensorten und der Schutz der bestehenden Agrobiodiversität eine zunehmend dringliche Notwendigkeit.

\footnotetext{
Batur/Dedeurwaerdere 2014

vgl. Norton 1986

vgl. Gliessman 2007; Deliège/Neuteleers 2015

Cline 2012
} 
Um den im Agroökosystem entstehenden evolutionären Entwicklungen entgegenzuwirken, sind Pflanzenzüchter, wollen sie neue Sorten einsetzen, in hohem Maße auf die Agrobiodiversität angewiesen. ${ }^{10}$ Einige Experten führen an, dass die Kulturpflanzenvielfalt in situ erhalten bleiben muss und die Konservierung in Saatgutbanken nicht ausreicht, da sich Kulturpflanzen nur weiterentwickeln, wenn sie sich auf freiem Feld an neue Umweltstressfaktoren anpassen. ${ }^{11}$ Eine ex-situ-Konservierung kann daher evolutionäre Weiterentwicklungen nicht nutzen, könnte aber dennoch eine wichtige Rolle spielen, indem auf diese Weise Sorten, die aktuell bei den Landwirten keinen großen Anklang finden, für einen möglichen Einsatz in der Zukunft bewahrt werden. ${ }^{12}$

Nahrungsmittelsicherheit ist nicht das einzige Interesse der Befürworter einer biologischen Erhaltung. Es gibt ein großes Arsenal an natürlichen Pestiziden, die aus der großen Artenvielfalt der Tropen entstammen ${ }^{13}$ und von denen viele das Potenzial aufweisen, den »ökologischen Fußabdruck « der Landwirtschaft zu reduzieren.

\subsection{Agrobiodiversität als Gemeinschaftserbe der Menschheit}

Landwirtschaftliche Sorten entstanden durch intensive, kreative Bemühungen und systematische Beobachtungen, die über Jahrtausende hinweg durchgeführt wurden. Der innovative Einsatz erfolgte dabei in kleinen Schritten durch eine große Anzahl von Landwirten auf der ganzen Welt. Im Gegensatz zu einem großen Teil der industriellen Innovationen kann für traditionelle Landrassen kein formaler Immaterialguttitel erworben werden, da die etablierten Anreizsysteme (Patente und Pflanzenzüchterrechte) einen eindeutigen Erfindungsschritt fordern [oder aber die Neuzüchtungen müssen »nicht naheliegend « (non-obvious) sein, wie einige Gesetzgeber es formulieren] und außerdem die Bedingungen der Homogenität, Stabilität sowie Neuartigkeit erfüllt sehen wollen. ${ }^{14}$ Traditionelle Landrassen dagegen sind gekennzeichnet durch Heterogenität und eine genetische Zusammensetzung, die je nach jahreszeitlichen Umweltbedingungen Variationen aufweist. Wie bei den meisten Innovationsbestrebungen, die sich übermäßig auf Langlebigkeit und kumulative schrittweise Verbesserungen über einen langen Zeitraum stützen, funktionieren unsere etablierten Regelungen zum geistigen Eigentum als Anreizsysteme nur unzureichend für Landrassen.

In den frühen Achtzigerjahren des vergangenen Jahrhunderts trat die Ernährungs- und Landwirtschaftsorganisation der Vereinten Nationen (FAO) für die Saatgutvielfalt als gemeinsames Erbe der Menschheit ein und distanzierte sich damit von den auf Eigentumsrechte ausgerichteten Anreizsystemen. ${ }^{15}$ Dieses Konzept, so hofften damalige Experten, sollte zum effizientesten Einsatz genetischer Ressourcen zur Erreichung der Nahrungsmittelsicherheit führen. Die Länder des »Globalen Nordens« begrüßten die Idee, solange die aktuellen kommerziellen Saatgutsorten aus diesem gemeinsamen Pool ausgeschlossen blieben. Die Länder des »Globalen Südens« jedoch wollten keine Ressourcen zu einem Pool beisteuern, für den nicht alle Parteien gemäß ihren Fähigkeiten einen Beitrag leisten. Daher lehnten diese Ländergruppe das Prinzip eines gemeinsamen Erbes ab, solange sie keinen Zugang zu neu entwickelten Sorten bekämen, die mithilfe von Ressourcen aus den »genetischen Hotspots« gezüchtet

$0 \quad$ Kloppenburg 2005

11 Stone 1994

12 Wood/Lenne 1997

13 Khater 2012

14 Siehe Trade-related Aspects of Intellectual Property Agreement 1994, Art. 27.1 und International Convention for the Protection of New Varieties of Plants 1991, Art. 5.1

$15 \quad$ FAO 1983 
werden, die aufgrund ihrer geografischen Lage größtenteils unter ihre Rechtssprechung fallen. ${ }^{16}$ Das Gemeinschaftserbe-Prinzip wurde daher zum großen Teil zugunsten von Vereinbarungen über »Zugang zu genetischen Ressourcen und gerechter Vorteilsausgleich « (access and benefit sharing) aufgegeben, die auf der Grundlage der 1992 verabschiedeten Biodiversitäts-Konvention ausgehandelt wurden. ${ }^{17}$

Zwei Jahrzehnte später jedoch zeigt die Erfahrung, dass die Vereinbarungen über Zugang und gerechter Vorteilsausgleich die Erwartungen nicht erfüllt haben. ${ }^{18}{ }$ Genetische Hotspots « erstrecken sich häufig über Landesgrenzen hinweg und in vielen Ländern erschweren komplizierte Verfahren die Vergabe von Zugangsrechten. Durch mehrere zuständige Behörden innerhalb eines Landes werden Verhandlungen kostspielig und zeitaufwendig. ${ }^{19}$ Darüber hinaus garantiert das Beschaffen der Rechte bei der Regierung eines Landes noch nicht, dass die Gemeinschaft, die die betreffende Sorte betreut oder entwickelt hat, ihren gerechten Anteil am Handel bekommt. Die politischen Agenden lokaler Regierungen stimmen oft nicht mit den Interessen der indigenen Gemeinschaften überein, die viele der betroffenen Sorten entwickelt haben. Außerdem sind eine Reihe von Sorten auch außerhalb ihres Habitats in botanischen Gärten und Saatgutbänken weltweit verfügbar.

Im selben Zeitraum haben Landwirte die Vorzüge von Open-Source-Pflanzenzüchtungsprogrammen (wieder-)entdeckt. ${ }^{20}$ Darüber hinaus werden sich Entwicklungshilfeexperten immer mehr der Tatsache bewusst, dass Investitionen in Agrarforschung vielfach zu weit höheren Erträgen führen. ${ }^{21}$ Dies erweckt zwei Gegenpositionen. Für diejenigen, die landwirtschaftliche Forschung hauptsächlich profitorientiert betreiben, spielt das Konzept des geistigen Eigentums eine zentrale Rolle. Für andere wird es immer weniger akzeptabel, Zeit und Ressourcen mit Recherchen zur Ausübungsfreiheit und Lizenzen oder dem Aushandeln von Materialtransfervereinbarungen $\mathrm{zu}$ vergeuden, wenn es vorrangig um Nahrungsmittelsicherheit und den Kampf gegen den Welthunger geht.

Die zunehmende Vernetzung der Welt bietet viele neue Möglichkeiten der Zusammenarbeit, jedoch wird dieses Potenzial im Keim erstickt, wenn es von einem massiven Verwaltungsapparat behindert wird. Pflanzenzüchter sind sich der gewaltigen Kosten im Hinblick auf Zeit und Geld sehr wohl bewusst, die für die Sicherstellung der Ausübungsfreiheit anfallen, und reagieren mittlerweile darauf. Gegenwärtig lässt sich ein Perspektivenwechsel hin zur Agrobiodiversität als Allmendegut beobachten. Diese Position ist dabei zu einem großen Teil auf den Erfolg der Open-Source-Initiativen in der Softwareentwicklung und die (Wieder-)Entdeckung von Saatguttauschsystemen indigener Landwirte zurückzuführen. ${ }^{22}$ Eine große Zahl von Landwirten und Wissenschaftlern verurteilt die aktuelle Verschiebung hin zu propertiären Anreizsystemen, da sie die traditionellen Werte Offenheit (d.h. Möglichkeit einer universellen Verifizierung), Kommunalismus, Reziprozität und Freigabe von Informationen und Materialien ausgehöhlt habe. ${ }^{23}$

Allmendegut ist ein Konzept aus den Wirtschaftswissenschaften, das Güter beschreibt, von deren Nutzung Konsumenten nicht ausgeschlossen werden können, bei deren Nutzung jedoch eine Rivalität zwischen den Nutzern herrscht. Die Nutzung eines solchen Guts verringert also den Gesamtnutzen, den andere daraus ziehen können, und es ist nicht möglich, die Menschen daran zu hindern, dieses Gut zu nutzen (im Gegensatz zu öffentlichen Gütern). ${ }^{24}$ Ein Ausschluss der Nutzung ist nur möglich, wenn

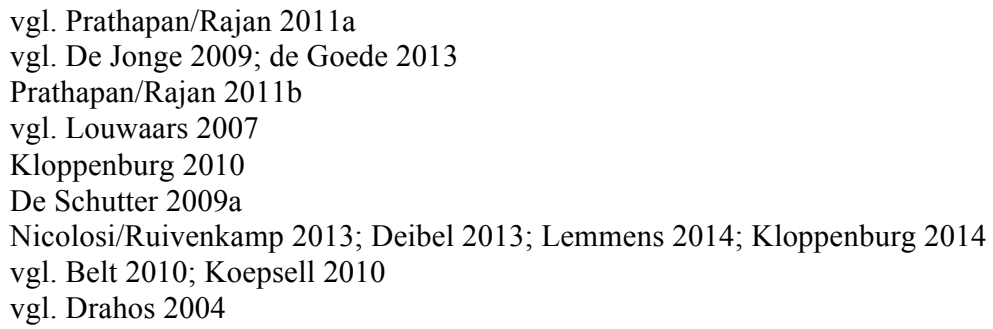


Eigentumsrechte an Saatgut allgemein anerkannt sind und durchgesetzt werden oder wenn es dem Pflanzenzüchter gelingt, die Elternlinien von Hybridsaaten geheim zu halten. ${ }^{25}$ Die Verbreitung einer Nahrungspflanzensorte beschleunigt ihre Anfälligkeit für Pathogene und verringert damit ihren Wert als landwirtschaftliche Ware.

Allmendegüter fallen in die Kategorie der unreinen öffentlichen Güter (common-pool resources). Klassische Beispiele hierfür sind Weiden, Fischgründe oder die Ozonschicht. ${ }^{26}$ Die Artenvielfalt bei Nahrungspflanzen kann als Allmendegut betrachtet werden und wirft ähnliche Managementprobleme auf wie die meisten anderen unreinen öffentlichen Güter. Da sich die Forschung zum Thema unreine öffentliche Güter stark weiterentwickelt hat seit Elinor Ostrom 1990 ihre bahnbrechende Arbeit dazu veröffentlichte, werden wir einige Ergebnisse der jüngeren Forschung verwenden, um in den nächsten Abschnitten ein neues Licht auf unabhängige Anreizsysteme für Saatzucht und Saatguterhaltung zu werfen.

\section{Marktanreize und gemeinsames Erbe der Menschheit}

Nachdem wir den aktuellen Stand der Dinge betrachtet haben, können wir uns nun fragen, ob das Konzept des gemeinsamen Erbes der Menschheit zu früh aufgegeben wurde. Innovationen in den Bereichen Konservierungstechniken und Informationsverarbeitungstechnologien haben die Argrarpraxis in hohem Maß verändert und die Grenzen des technologisch Machbaren erweitert. Hätte es Vorteile, das Konzept des gemeinsamen Erbes der Menschheit nach 30-jähriger Debatte wieder einzuführen? Was könnten Agrarpolitiker von Studien zu unreinen öffentlichen Gütern lernen, um das Konzept des gemeinsamen Erbes neu zu bewerten? Da die beiden Konzepte eng miteinander verwandt sind, lässt sich die Diskussion zum gemeinsamen Erbe durch die Literatur zur Steuerung unreiner öffentlicher Güter durchaus bereichern. Wir möchten im Folgenden einige erste Einsichten teilen.

\subsection{Das Gemeinschaftserbe-Prinzip der Menschheit}

Bevor wir die Wiedereinführung des Konzepts des Gemeinschaftserbes in Erwägung ziehen, müssen wir zwei Probleme anreißen, die im Laufe der letzten drei Jahrzehnte stark an Bedeutung gewonnen haben und die daher in unserer Evaluierung berücksichtigt werden müssen. Zum einen leben wir alle in einer Welt, in der Infrastruktur, Diskriminierung (wegen Geschlecht und ethnischer/kultureller Zugehörigkeit), Krankheiten und Umweltlasten sowie intellektuelles und finanzielles Kapital extrem ungleich verteilt sind. ${ }^{27}$ Zum anderen sind die Kosten für Forschung und Entwicklung stark gestiegen und Wissenschaftsagenden sind heute wesentlich stärker mit Marktanreizen verknüpft. ${ }^{28}$

Um die Möglichkeit einer Wiedereinführung des Prinzips des gemeinsamen Erbes auszuloten, werden wir zunächst untersuchen, was dieses Konzept ursprünglich verlangt und ihm in der Folge zentrale Kritikpunkte an Allmendegüter-Ressourcen gegenüberstellen. Auf diese Weise lässt sich feststellen, worauf bei der Erschaffung nicht-exklusiver Anreizsysteme zur Erhaltung der Agrobiodiversität zu achten

vgl. Kloppenburg 2005

Ostrom 2008

vgl. Singer 2004; Pogge 2008; Milanovic 2011

Stephan 2012 
ist. Frühe Arbeiten ${ }^{29}$ zum Begriff des gemeinsamen Erbes identifizierten die folgenden vier Ziele als zentral:

(1) Gemeinsames Management;

(2) Keine unilaterale Aneignung ohne die Verteilung des Nutzens auf alle;

(3) (Schnelle) Freigabe von Wissen aus wissenschaftlicher Forschung;

(4) Entmilitarisierung (bzw. keine schädliche Nutzung)

Bevor wir im Einzelnen auf diese Ziele eingehen, fassen wir sie für unsere konzeptuelle Untersuchung der Pflanzen-Genressourcen zu übersichtlicheren Kategorien zusammen. Unsere Diskussion konzentriert sich auf zwei Achsen. Im Hinblick auf das Management (1) sollten idealerweise Elemente, die als gemeinsames Erbe betrachtet werden, in einem System verwaltet werden, das im allgemeinen Konsens als demokratisch gelten kann und bei der konkreten Festlegung von Entscheidungsmechanismen gewisse Freiheiten ermöglicht. Die Punkte (2) und (3) erfordern beide, dass positive externe Effekte (d. h. materielle und immaterielle Vorteile) geteilt werden (falls möglich). Deswegen werden wir diese beiden Punkte gemeinsam behandeln. Was das vierte Ziel angeht, so werden wir diesen Punkt in unserer Erörterung überspringen, indem wir auf einen allgemein anerkannten Vertrag hinweisen, welcher den Einsatz von biologischen Kampfstoffen verurteilt: die Konvention über das Verbot der Entwicklung, Herstellung und Lagerung bakteriologischer (biologischer) Waffen und Toxinwaffen sowie über die Vernichtung solcher Waffen (1972).

\subsection{Gemeinsames Management}

In einem Managementsystem, das dem Prinzip des gemeinsamen Erbes der Menschheit untersteht, können Nutzer genetischer Ressourcen dieselben Probleme erwarten wie auch bei Allmendegütern, also Trittbrettfahrern, Schwierigkeiten bei der Koordinierung kollektiver Aktionen sowie Über- bzw. Unterbewirtschaftung. Im Falle der Agrobiodiversität sehen sie sich einem weiteren strittigen Element gegenüber, nämlich der Bewertung von Kulturpflanzen. Da bestimmte Pflanzensorten je nach Umweltbedingungen und Stufe der industriellen Entwicklung unterschiedlich bepreist werden, kann es zu Problemen bei der Entscheidungsfindung kommen. In dieser Hinsicht haben wir drei große Probleme des gemeinsamen Managements identifiziert, die stärkere negative Auswirkungen haben, wenn sehr ungleiche Partner die Ressourcen verwalten, sowie zusätzliche Probleme aufgedeckt, die sich auf die strukturelle Ähnlichkeit der Agrobiodiversität mit Allmendegütern zurückführen lassen.

Zunächst einmal erfordert die Erhaltung genetischer Ressourcen viel Arbeit. Falls die allgemeine Weltbevölkerung keinen Nutzen aus einer Ressource zieht, die sie aufbewahrt, können wir die langfristige Nachhaltigkeit dieser Bemühungen infrage stellen. ${ }^{30}$ Die Erhaltungslast dieser Ressourcen und die Fähigkeit, Vorteile aus solchen Bemühungen zu ziehen, sind gegenwärtig höchst ungleich verteilt, da eine solide wissenschaftliche und technologische Infrastruktur die Arbeit deutlich erleichtert und die Fähigkeit zur Vorhersage sowie Nutzung möglicher Vorteile verbessert.

Zweitens erfordert ein gemeinsames Management einen Konsens oder eine Übereinkunft zwischen den verschiedenen Parteien darüber, welchen Wert die einzelnen Pflanzensorten haben und welche Pflanzen bevorzugt werden sollten. Ein erstes Problem in dieser Hinsicht stellt sich dadurch, dass sich der Marktwert von Nahrungspflanzen deutlich von dem Wert jeder einzelnen Pflanzensorte für die Nahrungsmittelsicherheit unterscheidet. Darüber hinaus ist es schwierig, der erforderlichen Arbeit zur

29 vgl. Joyner 1986; Gorove 1972; Wolfrum 1983

30 Deliège/Neuteleers 2015 
Erhaltung einer Kulturpflanzensorte einen Wert zuzuweisen und festzulegen, wie die Arbeit im Zusammenhang mit dem Hinzufügen weiterer Sorten zum gemeinsamen Pool zu bewerten ist. Es müsste also ein Konsens über den Wert der Arbeit gefunden werden, die durch willentliche menschliche Bemühungen, durch die von Menschen gesteuerte Natur oder allein durch die Natur (durch Nichteinmischung) geleistet wird. Meinungsverschiedenheiten sind auch darüber zu erwarten, welcher Wert einer größeren Biodiversität zugemessen werden soll, die durch wissenschaftliche Durchbrüche oder stufenweise, kumulativ wirkende Bemühungen erreicht wird. ${ }^{31}$ Ein Allmendegut, das geschützt und weiterentwickelt werden muss, ist im Hinblick auf eine nachhaltige Nutzung großer Gefahr ausgesetzt, wenn die verschiedenen Nutzer die Beiträge der einzelnen Parteien höchst unterschiedlich bewerten. Zusätzlich variiert die Armut bedeutend je nach Breitengrad und Klimazone - ein Faktor, der zu einer großen Diskrepanz zwischen dem Bedarf der Armen an Nahrungspflanzen und den von den Reichen gewünschten Nahrungspflanzen führt. Insbesondere die tropischen Regionen müssen von der Agrarforschung dringend stärker beachtet werden. ${ }^{32}$ Darüber hinaus entsteht durch die finanziell besser gestellten Länder sowohl im »Globalen Norden« als auch im »Globalen Süden« eine gewaltige Nachfrage nach Nahrungspflanzen als Tierfutter und für den industriellen Einsatz. Die Kaufkraft dieser Gruppe schafft einen großen Anreiz, diese (nicht überlebenswichtige) Nachfrage zu erfüllen. Es ist eine bittere Wahrheit, dass europäische Kühe mehr Gelder in Form von Subventionen erhalten als ein Drittel der Weltbevölkerung in Form von Löhnen. ${ }^{33}$

Drittens bleibt zu überlegen, ob das Konzept eines gemeinsamen Managements ein ähnliches Problem aufwerfen wird wie wir es in der Biotechnologie beobachten: Die Existenz zu vieler Eigentümer (bzw. Akteure, die als solche handeln) könnte der sogenannten Tragik der Anti-Allmende Vorschub leisten. ${ }^{34}$ Unter Akteuren mit höchst divergierenden Interessen einen Konsens finden zu müssen, könnte Verhandlungen zum Erliegen bringen, auch wenn dies zum Nachteil aller Beteiligten geschähe.

Hinzu gibt es einige spezifische Probleme, die ein gemeinsames Management lösen müsste. An dieser Stelle bedienen wir uns der Erkenntnisse aus Elinor Ostroms Studien zur Steuerung von Allmendegütern. ${ }^{35}$ Ein gemeinsames Managementsystem müsste:

(1) klar definierte Grenzen setzen, um Verantwortungsbereiche zu etablieren, ohne ökologische Wechselbeziehungen zu beeinträchtigen,

(2) die sozialen und ökologischen Auswirkungen und Potenziale von Technologien beim Einsatz genetischer Materialien in Betracht ziehen,

(3) darauf achten, dass Konfliktlösungsmechanismen nicht durch das Ausdiskutieren von Problemen überlastet werden, die nichts oder wenig mit landwirtschaftlichen Genressourcen zu tun haben.

Die Festlegung, wer für das Management welcher Gruppen von Nahrungspflanzen zuständig ist, könnte viele der oben beschriebenen Schwierigkeiten umgehen. Es müsste ein System des gemeinsamen Managements gefunden werden, in dem Verantwortungsbereiche zugeschrieben und verteilt werden. Es gibt jedoch Grenzen, wie unabhängig voneinander diese Gruppen arbeiten können. Die Pflanzensorte, die eine Abteilung jeweils kontrolliert und steuert, gehört im Idealfall zu einem Ökosystem und lebt in Synergie mit anderen lebenden Organismen. ${ }^{36}$ Ein mangelndes Engagement einzelner Gruppen kann auch

\footnotetext{
vgl. Thompson 2010

De Schutter 2009b

Caparrós 2014

vgl. Heller 2008

Ostrom 1990

Gliessman 2007
} 
hier die aufmerksame Arbeit anderer Gruppen mindern, was einen zumindest grundlegenden regulatorischen Rahmen erforderlich macht.

Glücklicherweise gibt es in der Literatur eine Reihe von Quellen zu moralischer Verantwortung, die Licht auf diese komplexen Themen werfen und die Möglichkeit zur Unterscheidung geben. Das Nachdenken über die Verantwortung aus einer ethischen Perspektive heraus kann die Grundlage für einen fairen und effizienten regulatorischen Rahmen bilden. Tatsächlich sollten bei der Zuweisung von moralischer Verantwortung an verschiedene Akteure Fairness und Effizienz berücksichtigt werden, um individuelle und institutionelle Verantwortungsbereiche gleichmäßig zu verteilen. ${ }^{37}$ Ausgehend vom Begriff der Fairness betrachten wir hier das klassische Verständnis der moralischen Verantwortung, zu dem Schuldigkeit und Löblichkeit gehören, und ausgehend vom Begriff der Effizienz sehen wir das Ergebnis der Zuweisung moralischer Verantwortung, also welche Verteilung eher zu guten Ergebnissen führt.

Die Unterscheidung zwischen individueller und institutioneller Verantwortung ist in dem hier betrachteten Fall besonders wichtig, da Saatgut als ein intensiv gehandeltes und reguliertes Gut gilt. Zusätzlich ist es bedeutsam, von kollektiver moralischer Verantwortung zu sprechen, denn die Gesamtheit der Nahrungspflanzen, die die Agrobiodiversität ausmachen, ist ein Allmendegut, das im Idealfall einem gemeinsamen Management unterliegt. Da verschiedene Autoren jeweils auf ihre eigene Weise zwischen diesen Begriffen unterscheiden, schlagen wir an dieser Stelle Seamus Millers Definition vor, der eine Gemeinschaftsrechnung individueller moralischer Verantwortung für eine kollektive Aktion anregt. ${ }^{38}$ Auf diese Weise hat jeder Akteur eine moralische Verantwortung, die mit der moralischen Verantwortung anderer Akteure vernetzt ist, um eine erwünschte Sachlage zu erreichen. Dabei ist es unerheblich, ob diese Akteure Individuen oder Institutionen sind. Ohne im Detail auf die Mechanismen der kollektiven moralischen Verantwortung einzugehen, soll es im Rahmen dieses Themas reichen, die Bedeutung dieser Vernetzung zu unterstreichen und darauf hinzuweisen, dass es Werkzeuge gibt (nützliche analytische Unterscheidungen), mit deren Hilfe sich das komplexe Feld jener Akteure, die sich mit der Förderung der Agrobiodiversität beschäftigen, darstellen lässt.

$\mathrm{Zu}$ guter Letzt kann die moralische Verantwortung auf viele unterschiedliche Arten klassifiziert werden. ${ }^{39}$ Die Aufstellung einer Typologie der moralischen Verantwortung ist nützlich, um das aktuelle System zu beschreiben. Eine solche Typologie hilft auch bei der Festlegung, welche Art von Verantwortung die verschiedenen Akteure übernehmen. Die Art könnte dabei unter den betroffenen Akteuren variieren, um auf diese Weise gemeinsam das Ziel zu erreichen, nämlich die Erhaltung der Agrobiodiversität. Kürzlich wurde vorgeschlagen, moralische Verantwortung für Gefährdungen auf der Grundlage von Besitzansprüchen auf das Saatgut zuzuschreiben. ${ }^{40}$ Im Fall der Agrobiodiversität haben Saatgutbesitzer die moralische Verantwortung, diese nicht negativ zu beeinflussen; dies ließe sich ausweiten zu einer moralischen Verantwortung, die Diversität in der Landwirtschaft zu fördern sowie extrem weitflächige Monokulturen zu verhindern.

Außerdem sollte berücksichtigt werden, dass technologische Innovation die Erhaltungsarbeit in hohem Maße erleichtern kann. ${ }^{41}$ Es müssen Kompromisse zwischen Technologieherstellern und Ursprungsländern von genetischen Ressourcen eingegangen werden, um sicherzustellen, dass jeder sich nach seinen Fähigkeiten am Gemeinschaftspool beteiligt. Die Menschheit als Ganzes, so die Hoffnung, wird davon profitieren, dass genetische Ressourcen mithilfe arbeitssparender und wirksamer Technologien von vielen freigegeben und bewahrt werden; das zur Verfügungstellen von Technologien sowie das wissenschaftliche

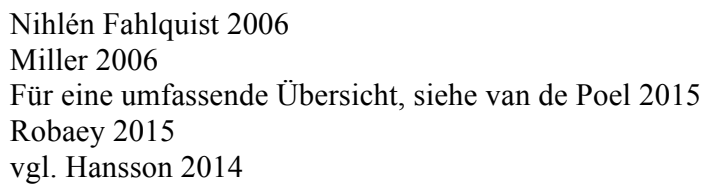


und technische Expertenwissen werden darüber hinaus die Fähigkeit finanziell armer Länder zur Bewertung und Erhaltung der genetischen Ressourcen unter ihrer Rechtssprechung verbessern.

Die Zivilgesellschaft, so unser Votum, muss größere Anstrengungen unternehmen, damit Entscheidungsorgane, die einen erfolgreichen Austausch landwirtschaftlicher Genressourcen garantieren sollen, nicht regelmäßig für andere Zwecke benutzt werden. Dies wird eine große Herausforderung darstellen, da viele Nationen ihre Konflikte zurückstellen werden müssen, um sicherzustellen, dass die gemeinsame Sorge um die Nahrungsmittelsicherheit angemessen angegangen wird.

\subsection{Verbot unilateraler Aneignung und Vorteilsausgleich}

Zeitgemäße oder neue Anwendungen für genetische Ressourcen zu finden und zu entwickeln, erfordert einen beträchtlichen Ressourceneinsatz. Exklusive Rechte können dabei helfen, Forschung und Entwicklung zu beschleunigen - ein wichtiger Faktor, den die Menschheit in einer Zeit, in der viele dieser genetischen Ressourcen vom Verschwinden bedroht sind, nicht vernachlässigen sollte.

Geistiges Eigentum als Werkzeug zur Beschleunigung von Innovation ist mit einigen bedeutenden sozialen und wirtschaftlichen Kosten verbunden. Es ist ein teures System, erfordert juristisches Expertenwissen, schränkt anfangs die Transparenz ein (vor allem vor der Patenteinreichung), ist ein langsamer Prozess und ermöglicht bis zu einem gewissen Umfang die Kontrolle über nachfolgende Innovationen. Länder mit einer großen Zahl ausgebildeter Anwälte, einem soliden Finanzsystem und einer etablierten Forschungs- und Entwicklungsbranche können in einem solchen System von einem deutlichen Vorsprung profitieren. ${ }^{42}$ Wo die Bedürfnisse unterschiedlich sind und die Nutzungskapazitäten so verschieden ausfallen, ist zu erwarten, dass das Zurückgreifen auf einen gemeinsamen Pool zu gewaltigen Unterschieden hinsichtlich der

»Ausbeutungsmöglichkeiten « führt, und dies selbst dann, wenn die Ergebnisse der Innovation allen zugänglich sind. Um ein Beispiel zu nennen: Die Entwicklung einer ertragreichen Maissorte, die sich in gemäßigten Klimazonen anbauen lässt, aus gemeinsam verwalteten genetischen Ressourcen bringt für nördliche Länder (und Länder ganz im Süden) große Vorteile, während sie für die Maisbauern aus den tropischen und subtropischen Regionen nur zu einer Verschärfung der Marktkonkurrenz führen, der sie sich ohnehin gegenübersehen.

Die unilaterale Aneignung ist nicht das einzige Problem. Selektiver Einsatz und damit selektive Erhaltung kann für nachfolgende Generationen ebenso problematisch werden wie vorübergehende Exklusivität. Landwirte, öffentliche Institute und Saatgutunternehmen können sich aus höchst unterschiedlichen Motiven darum bemühen, bestimmte Sorten zu erhalten und weiter zu züchten. ${ }^{43}$ Gründer von Saatgutbanken haben ebenfalls ganz eigene Motive. Die Gründe, um Saatgut zu erhalten, variieren auch in Abhängigkeit von verschiedenen Belastungen, denen die einzelnen Interessenvertreter ausgesetzt sind. Marktkräfte, Nachfrage nach Nahrungsmitteln, Veränderungen der Umwelt und ästhetische Präferenzen sind Variablen, die Konservierungsentscheidungen formen. Die Möglichkeit, eine größere Auswahl an Saatgutsorten (auf Feldern oder in Saatgutbanken) zu verwenden, ist abhängig von den Einschränkungen und Gelegenheiten in den jeweiligen Ländern. Welches Saatgut zur unmittelbaren Verwendung oder für die Erhaltung ausgewählt wird, hängt von den Ressourcen der betroffenen Partei ab und von der Freiheit, die Landwirte haben, wenn sie Saatgut für die Aussaat wählen. Der Erhalt eines gemeinsamen Erbes kann also höchst instrumentalen Motiven unterliegen.

42 Timmermann/van den Belt 2012

43 Wood/Lenne 1997 
Aus dem Blickwinkel der globalen Gerechtigkeit müsste auch das Problem der Länder, die sowohl an Finanzmitteln wie auch an Agrobiodiversität arm sind, berücksichtigt werden. Zwar befinden sich landwirtschaftliche »genetische Hotspots« vorwiegend im »Globalen Süden«, jedoch bedeutet dies nicht, dass dieser Reichtum gleichmäßig auf alle Länder des »Globalen Süden« verteilt wäre. Das Gemeinschaftserbe-Prinzip bietet den an landwirtschaftlichen genetischen Ressourcen armen Ländern des "Globalen Südens" hierbei bedeutende Vorteile. Marktanreize dagegen sind für diese Gruppe mit wesentlichen Nachteilen verbunden. Der Mangel an finanziellem und biologischem Kapital bringt sie in eine prekäre Verhandlungsposition, was die Erfüllung des Bedarfs an genetischen Ressourcen für ihre Nahrungsmittelsicherheit angeht.

Dennoch, so unser Votum, sollte man bei Reflexionen über die Verteilungsgerechtigkeit bei allem Streben nach Gerechtigkeit unbedingt darauf achten, weder langfristige Gelegenheiten zu versäumen noch (zukünftige) Ressourcen zu vergeuden. Das Beharren auf einem völlig gerechten System könnte zur Folge haben, dass Gelegenheiten verstreichen, neue Saatgutsorten zu entwickeln und den schrumpfenden Agrobiodiversitätspool rechtzeitig zu nutzen. In einer Welt, in der genetische Ressourcen in hohem Tempo verschwinden, müssen auch zweitbeste Anreizsysteme auf ihre Fähigkeit hin untersucht werden, rechtzeitig Innovationen hervorzubringen. Durch den raschen Verlust der Agrobiodiversität könnte die allgemeine Bevölkerung sich verpflichtet sehen, ein System zu unterstützen, das im Hinblick auf die Gerechtigkeit gewisse Defizite aufweist, jedoch in einem früheren Stadium implementiert werden kann. Ein solches System ließe sich, sobald es erst einmal etabliert ist, im Laufe der Zeit noch verbessern.

Auf der anderen Seite sollte eine vorübergehende Exklusivität aber auch nicht allzu voreilig begrüßt werden. Insbesondere im Hinblick auf Arzneimittel gibt es eine Reihe von Vorschlägen, die einen unmittelbaren Zugang ermöglichen könnten. Zu denken wäre in diesem Zusammenhang an Preissysteme ${ }^{44}$ oder eine Variation des Health Impact Funds ${ }^{45}$. Ein zentraler Fonds könnte das Problem des Zugangs zu landwirtschaftlicher Innovation und Forschungsdaten lösen und dabei gleichzeitig die notwendigen finanziellen Anreize für Innovationen bieten.

Bei der Beurteilung von Anreizsystemen muss untersucht werden, wie Unterschiede zwischen fairen und gleichen Anteilen (z. B. pro Kopf) sich mit dem Prinzip des gemeinsamen Erbes vereinbaren lassen. Wollen einzelne Akteure in Zahlen ausdrücken, was genau ein »fairer Anteil« ist, stellt sich das Problem der unterschiedlichen Bewertung - ein Konfliktfaktor, der im Zusammenhang mit den Erfahrungen mit Vereinbarungen über Zugang $\mathrm{zu}$ genetischen Ressourcen und im Kontext eines gerechten Vorteilsausgleichs bereits gut dokumentiert ist. ${ }^{46}$

Dies lässt offen, wie viele Betriebskosten abgezogen werden können, bevor Erträge geteilt werden. Dieser Punkt muss im Besonderen geregelt werden, wenn eine große Anzahl privater Unternehmen die Agrobiodiversität mit modernen wissenschaftlichen Methoden und Technologien profitorientiert verwertet. Auch bleibt unklar, inwieweit die Kosten, mit denen negative externe Effekte abgefangen werden, die durch die Ausnutzung von Ressourcen entstehen, geteilt werden sollten oder ob die sogenannten Opportunitätskosten der Forschungskonzernen ebenfalls abgesetzt werden können. Was die Inklusivität der Forschung betrifft, führen einige Autoren aus, dass die wissenschaftliche Forschung an Ressourcen, die als gemeinsames Erbe gelten, für Wissenschaftler des »Globalen Südens« auch Kapazitätsaufbau bieten sollte, um ihnen die Teilnahme an der globalen Wissenschaftsgemeinde zu ermöglichen. ${ }^{47}$

44 Love/Hubbard 2007

45 Hollis/Pogge 2012 und vgl. Timmermann/van den Belt 2012

46 Wynberg/Schroeder/Chennells 2009

47 vgl. Shaver 2010; Shaheed 2012; Timmermann 2014 
Zum Abschluss wollen wir unsere Aufmerksamkeit der Idee der »Gemeinsamkeit« zuwenden. Wenn über ein gemeinsames Erbe gesprochen wird, wem ist dann dieses Erbe gemeinsam? Während diese Frage immer noch in hohem Maße strittig ist, gibt es eine Reihe von Bedürfnissen und Werten, die alle Menschen gemeinsam teilen, ${ }^{48}$ auch wenn die Dringlichkeit, mit der diese Bedürfnisse auftreten, stark variiert. Zwar haben alle Menschen dieselben Grundbedürfnisse, jedoch ist jeder Einzelne je nach geografischer Position, Haushaltseinkommen, sanitärer Infrastruktur und Exposition gegenüber Krankheiten an unterschiedlichen Stellen angreifbar. So tragen die Tropen eine deutlich höhere Krankheitslast als andere Gebiete. Abwasserinfrastruktur und Kühlketten für Lebensmittel verhindern eine Vielzahl von Krankheiten und verringern den Vitamin- und Mineralstoffverlust, der durch Durchfall und andere Erkrankungen verursacht wird. Ernährung und Gesundheit sind eng miteinander verknüpft. Menschen, die anfälliger für Erkrankungen sind, müssen besonders auf ihre Ernährung achten, um gesund zu bleiben. In verschiedenen Lebensabschnitten hat der Mensch jeweils einen unterschiedlichen Nahrungsbedarf. Länder mit alternder Bevölkerung haben insgesamt daher einen anderen Ernährungsbedarf als Länder mit einer sehr jungen Bevölkerung.

Ist es trotzdem sinnvoll, von einem gemeinsamen Erbe zu sprechen, wenn Lasten und Dringlichkeit der Bedürfnisse weltweit so ungleich auf die verschiedenen sozialen Gruppen verteilt sind? Während einige Menschen Nahrungsmittel hauptsächlich als Brennstoff sehen bzw. sehen müssen, betrachten andere Essen als zentrales Element in ihrem Leben und verlangen von landwirtschaftlichen Erzeugnissen nicht nur die Befriedigung physiologischer Bedürfnisse, sondern auch komplexe Geschmackserfahrungen oder die Fortführung kultureller Traditionen. ${ }^{49}$ Diese unterschiedlichen Bedürfnisse und/oder Präferenzen spiegeln auch die Art von Erbe wider, das die Menschheit als Ganzes bewahren wird. Ein Teil der Menschheit wird Nahrungspflanzen aufgrund der Nahrungsmittelsicherheit auswählen, ein anderer wird sich bemühen, neue Nahrungspflanzensorten zu erhalten und weiterzuzüchten, um das kulinarische Welterbe zu erhalten und weiterzuentwickeln.

Trotz dieser Unterschiede ist allen Menschen immer noch das Bedürfnis gemein, sich zu ernähren, sowie der Drang, souverän darüber zu entscheiden, wie sie dieses Bedürfnis befriedigen. ${ }^{50} \mathrm{Ob}$ die Landwirtschaft nun der Befriedigung eines Grundbedürfnisses dient oder der Erzeugung wohlschmeckenderer Gemüsesorten - die Landwirte kämpfen zunehmend um Souveränität in der Entscheidung, wie sie ihre Felder bestellen. Trotz aller Diskrepanzen zwischen Arm und Reich besteht noch immer ein universelles Bedürfnis, die Mittel zum Weiterzüchten von Pflanzen nach individuellen Wünschen und Erfordernissen wieder in Besitz zu nehmen. Nahrungsmittelsouveränität benötigt einen freieren Zugang zu genetischen Ressourcen. Bauer und Pflanzenzüchter die einen ungenügenden Zugang zu diesen Ressourcen haben, fehlt ein essentieller Mittel zur Weiterzüchtung von Nutzpflanzen. Ein Übermaß an Privatisierung genetischer Ressourcen durch Biopatente und andere Formen geistigen Eigentums erschwert Kleinbauern, Start-up-Unternehmen sowie Institute in ärmeren Ländern und Regionen autonome Pflanzenzuchtprogramme; hierin liegt ein Problem, das institutionelle Vielfalt und Artenvielfalt verringert. ${ }^{51}$

48 vgl. Beitz 2009; Sen 2009

49 vgl. Korthals 2004

50 vgl. Beuchelt/Virchow 2012;Timmermann/Félix 2015

51 Danksagung: CT erhielt eine Förderung des Jacques Loeb Centre for the History and Philosophy of the Life Sciences an der Ben-Gurion-Universität des Negev und des Förderprogramms für Postdoktoranden der Universidad Nacional Autónoma de México. ZR erhielt Mittel im Rahmen des Forschungsprogramms New Technologies as Social Experiments, das von der Nederlandse Organisatie voor Wetenschappelijk Onderzoek (Niederländischen Organisation für wissenschaftliche Forschung, NWO) unter der Bewilligungsnummer 016.114.625 unterstützt wird. Eine frührere 


\section{Literatur}

Batur, Fulya/Dedeurwaerdere, Tom (2014): The Use of Agrobiodiversity for Plant Improvement and the Intellectual Property Paradigm: Institutional Fit and Legal Tools for Mass Selection, Conventional and Molecular Plant Breeding, in: Life Sciences, Society and Policy 10:14

Beitz, Charles (2009): The Idea of Human Rights.

Belt, Henk van den (2010): Robert Merton, Intellectual Property, and Open Science, in: Radder, Hans (Hrsg.), The Commodification of Academic Research : Science and the Modern University. $187 \mathrm{ff}$.

Beuchelt, Tina D./Virchow, Detlef (2012): Food Sovereignty or the Human Right to Adequate Food: Which Concept Serves Better as International Development Policy for Global Hunger and Poverty Reduction?, in: Agriculture and Human Values 29(, $259 \mathrm{ff}$.

Caparrós, Martín (2014): El Hambre.

Cline, William R. (2012): Global Warming and Agriculture: Impact Estimates by Country.

De Goede, Ludo (2013): From Common Heritage to Intellectual Property: A Global Justice Perspective on Shifting Property Regimes for Plant Genetic Resources.

Deibel, Eric (2013): Open Variety Rights: Rethinking the commodification of plants, in: Journal of Agrarian Change 13, 282 ff.

De Jonge, Bram (2009): Plants, Genes and Justice: An Inquiry into Fair and Equitable Benefit-Sharing. Wageningen, Disseration.

Deliège, Glenn/Neuteleers, Stijn (2015): Should Biodiversity Be Useful?, in: Environmental Values 24, $165 \mathrm{ff}$.

De Schutter, Olivier (2009a): International Trade in Agriculture and the Right to Food.

De Schutter, Olivier (2009b): Seed Policies and the Right to Food: Enhancing Agrobiodiversity and Encouraging Innovation (Report Presented to the UN General Assembly, 64th Session, UN Doc. A/64/170).

Drahos, Peter (2004): The Regulation of Public Goods, in: Journal of International Economic Law 7, $321 \mathrm{ff}$.

FAO (1983): International Undertaking on Plant Genetic Resources for Food and Agriculture.

Gliessman, Stephen R (2007): Agroecology: The Ecology of Sustainable Food Systems.

Gorove, Stephen (1972): The Concept of Common Heritage of Mankind: A Political Moral Or Legal Innovation, in: San Diego Law Review 9, 390 ff.

Hansson, Sven Ove (2014): Agricultural Biotechnology for Health and the Environment, in: Ahuja, M. R./Ramawat, KishanGopal (Hrsg.), Biotechnology and Biodiversity. $67 \mathrm{ff}$.

Heller, Michael (2008): The Gridlock Economy: How Too Much Ownership Wrecks Markets, Stops Innovation, and Costs Lives.

Heyd, David (2010): Cultural Diversity and Biodiversity: A Tempting Analogy, in: Critical Review of International Social and Political Philosophy 13, $159 \mathrm{ff}$.

Hollis, Aidan, and Thomas W. Pogge (2012):The Health Impact Fund: Making New Medicines Accessible for All.

Joyner, Christopher C. (1986): Legal Implications of the Concept of the Common Heritage of Mankind, in: The International and Comparative Law Quarterly 35, $190 \mathrm{ff}$.

Khater, HanemFathy (2012): Prospects of Botanical Biopesticides in Insect Pest Management, in: Pharmacologia 3, $641 \mathrm{ff}$.

Kloppenburg, Jack R. (2005):First the Seed: The Political Economy of Plant Biotechnology. 2. Auflage.

Kloppenburg, Jack R. (2010): Impeding Dispossession, Enabling Repossession: Biological Open Source and the Recovery of Seed Sovereignity, in: Journal of Agrarian Change 10, $367 \mathrm{ff}$.

Kloppenburg, Jack R. (2014): Re-Purposing the Master's Tools: The Open Source Seed Initiative and the Struggle for Seed Sovereignty, in: Journal of Peasant Studies 41, $1225 \mathrm{ff}$.

Kloppenburg, Jack R./Kleinman, Daniel Lee (1987): The Plant Germplasm Controversy, in: BioScience 37, 190 ff.

Version dieser Arbeit wurde im Oktober 2014 im Rahmen der Klausurwoche vorgestellt. Wir möchten den Seminarteilnehmern für ihre wertvollen Anmerkungen danken. 
Koepsell, David (2010): Back to Basics: How Technology and the Open Source Movement Can Save Science, in: Social Epistemology 24, $181 \mathrm{ff}$.

Korthals, Michiel (2004): Before Dinner: Philosophy and Ethics of Food.

Lacy, William B. (1994): Biodiversity, Cultural Diversity, And Food Equity, in: Agriculture and Human Values 11, $3 \mathrm{ff}$.

Lemmens, Pieter (2014): Re-Taking Care: Open Source Biotech in Light of the Need to Deproletarianize Agricultural Innovation, in: Journal of Agricultural and Environmental Ethics27, $127 \mathrm{ff}$.

Louwaars, Niels (2007): Seeds of Confusion: The Impact of Policies on Seed Systems. Wageningen, Disseration.

Love, James/Hubbard, Tim (2007): The Big Idea: Prizes to Stimulate R\&D for New Medicines, in: Chicago-Kent Law Review 82, $1519 \mathrm{ff}$.

Malaterre, Christophe (2013): Microbial Diversity and the 'lower-Limit' Problem of Biodiversity, in: Biology \& Philosophy 28, $219 \mathrm{ff}$.

Milanovic, Branko (2011): Worlds Apart: Measuring International and Global Inequality.

Miller, Seamus (2006): Collective Moral Responsibility: An Individualist Account, in: Midwest Studies in Philosophy 30, $176 \mathrm{ff}$.

Nicolosi, Guido/Ruivenkamp, Guido (2013): Re-Skilling the Social Practices: Open Source and Life-Towards a CommonsBased Peer Production in Agro-Biotechnology?, in:Science and Engineering Ethics 19, $1181 \mathrm{ff}$.

Nihlén Fahlquist, Jessica (2006): Responsibility Ascriptions and Public Health Problems, in: Journal of Public Health 14, 15 ff.

Norton, Bryan G. (1986): Conservation and Preservation: A Conceptual Rehabilitation, in: Environmental Ethics 8, 195 ff.

Ostrom, Elinor (1990): Governing the Commons: The Evolution of Institutions for Collective Action.

Ostrom, Elinor (2008): The Challenge of Common-Pool Resources, in: Environment: Science and Policy for Sustainable Development 50, $8 \mathrm{ff}$.

Pogge, Thomas W. (2008): World Poverty and Human Rights: Cosmopolitan Responsibilities and Reforms. 2. Auflage.

Prathapan, K Divakaran/Rajan, Priyadarsanan Dharma (2011a): Biological Diversity: A Common Heritage, in: Economic \& Political Weekly 46, $15 \mathrm{ff}$.

Prathapan, K Divakaran/Rajan, Priyadarsanan Dharma (2011b): Biodiversity Access and Benefit-Sharing: Weaving a Rope of Sand, in: Current Science 100, $290 \mathrm{ff}$.

Robaey, Zoë (2015): Looking for Moral Responsibility in Ownership: A Way to Deal with Hazards of GMOs, in: Journal of Agricultural and Environmental Ethics 28, $43 \mathrm{ff}$.

Sen, Amartya (2009): The Idea of Justice.

Shaheed, Farida (2012): Report of the Special Rapporteur in the Field of Cultural Rights. United Nations (A/HRC/20/26).

Shaver, Lea (2010): The Right to Science and Culture, in: Wisconsin Law Review 121, $121 \mathrm{ff}$.

Singer, Peter (2004): One World: The Ethics of Globalization.2. Auflage.

Stephan, Paula E. (2012): How Economics Shapes Science.

Stone, Christopher D. (1994): What To Do About Biodiversity: Property Rights: Public Goods, and the Earth's Biological Riches, in: Southern California Law Review 68, $577 \mathrm{ff}$.

Thompson, Paul B (2010): Is Our Agricultural Technology Innovation System Up to 21st Century Challenges?, in: Science Progress 24 .

Timmermann, Cristian (2014): Sharing in or Benefiting from Scientific Advancement?" Science and Engineering Ethics 20, $111 \mathrm{ff}$.

Timmermann, Cristian/Félix, Georges F. (2015): Agroecology as a Vehicle for Contributive Justice, in: Agriculture and Human Values 32, $523 \mathrm{ff}$.

Timmermann, Cristian/Belt, Henk van den (2012): Global Justice Considerations for a Proposed 'climate Impact Fund, in: Public Reason 4, 182 ff.

Tomich, Thomas P./Brodt, Sonja/Ferris, Howard/Galt, Ryan/Horwath, William R./Kebreab, Ermias/Leveau, Johan H.J./Liptzin, Daniel/ Lubell, Mark/Merel, Pierre (2011): Agroecology: A Review from a Global-Change Perspective, in: Annual Review of Environment and Resources 36, $193 \mathrm{ff}$.

Van de Poel, Ibo (2015): Moral Responsibility, in: van de Poel, Ibo/Royakkers, Lamber/Zwart, Sjoerd D. (Hrsg.), Moral Responsibility and the Problem of Many Hands, $12 \mathrm{ff}$. 
Wolfrum, Rüdiger (1983): The Principle of the Common Heritage of Mankind, in: Heidelberg Journal of International Law 43, $312 \mathrm{ff}$.

Wood, David/Lenne, Jillian M. (1997): The Conservation of Agrobiodiversity on-Farm: Questioning the Emerging Paradigm, in: Biodiversity \& Conservation 6, $109 \mathrm{ff}$.

Wynberg, Rachel/Schroeder, Doris/Chennells, Roger (Hrsg.) (2009): Indigenous Peoples, Consent and Benefit Sharing: Lessons from the San-Hoodia Case. 